\title{
Predominancia del autoconcepto en estudiantes con conductas antisociales del Callao
}

\section{Predominance of Self-concept in Students with Antisocial Behavior of Callao}

\author{
Jessica Palacios-Garay* \\ Universidad Nacional Federico Villarreal, Lima, Perú \\ ORCID: https://orcid.org/0000-0002-2315-1683 \\ José Coveñas-Lalupú \\ Universidad Nacional Federico Villarreal, Lima, Perú \\ ORCID: https://orcid.org/0000-0001-5318-9618
}

Recibido 12-08-18 Revisado 15-09-18 Aprobado 04-01-19 En línea 10-01-18

*Correspondencia

Email: jpalaciosg@unfv.edu.pe

\section{Citar como:}

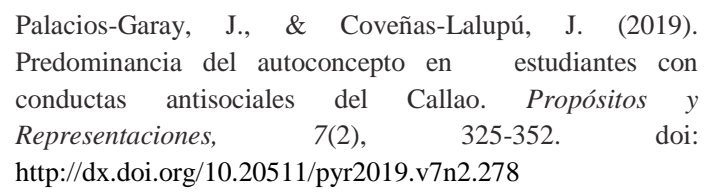




\section{Resumen}

El objetivo de la investigación fue determinar la dimensión predominante que más influye en el autoconcepto en estudiantes con conductas antisociales. El trabajo en mención es de tipo sustantiva, de un diseño no experimental de corte transversal. El método empleado fue el hipotético deductivo. La muestra estuvo conformada por 98 adolescentes con conductas antisociales, el muestreo utilizado fue el no probabilístico intencional. El instrumento utilizado fue AF-5 de García y Musitu (1999). Los resultados indicaron que el autoconcepto que influye en las adolescentes fue el autoconcepto emocional

Palabras clave: Autoconcepto; Académico; Social, Emocional; Familiar y físico.

\section{Summary}

The objective of the research was to determine the predominant dimension that has more influence over the self-concept in students with antisocial behaviors. This work is of a substantive type, of a non- experimental cross-sectional design. The method used was the hypothetical deductive. The sample consisted of 98 adolescents with antisocial behavior, the sampling used was intentional non- probabilistic. The instrument used was AF-5 by Garcia and Musitu (1999). The results indicated that the self-concept that influences adolescents was emotional self-concept.

Keywords: Self-Concept; Academic; Social; Emotional; Family and Physical

\section{Introducción}

El autoconcepto es una representación mental que los individuos tienen de sí mismos. Así como los individuos tienen representaciones mentales del mundo que los rodea, de otras personas y eventos significativos, también tienen representaciones de sí mismos. Para construir un autoconcepto la gente pone atención a la retroalimentación que recibe en la vida cotidiana que revelan sus atributos, características y preferencias personales.

El autoconcepto juega un papel decisivo y central en el desarrollo de la personalidad, tal como lo destacan las principales teorías psicológicas; un autoconcepto positivo está en la base al buen funcionamiento personal, social y profesional. De ahí que lograr un autoconcepto positivo sea uno de los objetivos más pretendidos en numerosos programas de intervención psicológica (educativa, clínica, comunitaria, cívica) para los que se demandan estrategias y recursos que permitan su mejora.

De acuerdo con García y Musitu (1999) el autoconcepto puede entenderse como la percepción que el individuo tiene de sí mismo, basado en sus experiencias con los demás y en las atribuciones de su propia conducta. Involucra componentes emocionales, sociales, físicos y académicos. Se trata de una configuración organizada de percepciones de sí mismo, admisible a la conciencia y al conocimiento. Es un esquema cognitivo muy complejo construido a través de experiencias previas con respecto al mundo que lo rodea.

En los últimos años se ha observado que el ámbito educativo regular y más aún en los centros de rehabilitación social viene presentando diversas dificultades y problemas en las habilidades sociales que manifiestan los estudiantes que presentan un bajo o limitado nivel de autoestima, inseguridad al comunicarse, poca tolerancia a la frustración una fuerte carga de agresividad e ira, y un lenguaje coprolálico. 
Es importante destacar que en estas instituciones de rehabilitación social las diversas estudiantes o internas presentan un gran historial de conductas antisociales ya que muchas de ellas vienen de una situación de extrema pobreza, prostitución, abandono, consumo de drogas y actos delictivos; situaciones estas que han menoscabado su autoconcepto y seguridad personal creando en ellas inestabilidad, baja tolerancia a la frustración y otros componentes más sociales de riesgo. Por lo que se hace necesario que desde un punto de vista educativo debería desarrollarse el autoconcepto de estas internas que determinen una confianza y seguridad para su rehabilitación social y con ellos su reinserción en el campo laboral y familiar situación indispensable dado que la totalidad de estas internas se encuentran en una etapa crítica y de transición como es la adolescencia situación que las hace muy vulnerables para la situación de riesgo social.

\section{Autoconcepto.}

Kimmel y Weiner (1998, citado en Schmidt, 2010) sostuvieron que "el autoconcepto es la opinión que uno tiene de su persona y es un conjunto de ideas y sentimientos que uno tiene de sí mismo" (p.96).

El autoconcepto es "la imagen del yo-conocido que tiene cada persona. Es decir, la construcción mental de cómo se percibe a sí misma; incluye valoraciones de todos los parámetros que son relevantes para la persona: desde la apariencia física hasta las capacidades" sociales, intelectuales, etc.

Cardenal (1999) consideró que el autoconcepto son un conjunto de opiniones descriptivas y evaluativas sobre sí mismo y en él se manifiesta de la forma en las que las personas se representan, se conocen se valoran.

La definición más sustantiva es la propuesta por Byrne y Baron (2005), quienes afirman que es la opinión que se tiene de sí, son las actitudes, emociones y el conocimiento con relación a las capacidades y aceptación social (p. 169).

Por su parte, Cerviño (2008) afirmó que:

El autoconcepto no nace con las personas, por el contrario, se edifica y define en el trayecto de toda la vida y está influida por las diversas experiencias que las personas presentan en medio de su ámbito escolar, social y familiar que haya vivido. (p. 34).

Antes de los años 80, la mayoría de los estudios relacionados con el autoconcepto estaban basados en una perspectiva unidimensional del mismo (Harter, 1990), pero es a partir de esta fecha cuando el modelo multidimensional es el utilizado frecuentemente para investigar dicho constructo. Por lo tanto, si la mayoría de los instrumentos utilizados para evaluar el autoconcepto antes de los ochenta, estaban basados en una estructura unidimensional del mismo, a partir de esta fecha los instrumentos elaborados para evaluarlo parten siempre de la multidimensionalidad que definió ineludiblemente a está aspecto de la personalidad (Byrne, 1996). Es interesante considerar los siete modelos teóricos que sirven para acercarse a delimitar el autoconcepto, según la posición de Byrne (1996). 
Modelos unidimensionales.

\section{Modelo nomotético.}

Es el modelo más antiguo, su creador fue Soares y Soares (1983), que entendió el autoconcepto desde una perspectiva muy generalista y universal. Algunos autores como Marsh y Hattie (1996) lo expresaron como el modelo del "factor general", lógicamente con ámbito unidimensional. Así el autoconcepto general sería la suma de los diferentes tipos de autoconcepto: familiar, escolar, social, etc. Coopersmith (1967) defendió este modelo porque le permitía valorar el autoconcepto de los sujetos hasta la pre-adolescencia, ya que hasta que un individuo alcanza está edad le resulta difícil lograr una autodescripción de las diferentes dimensiones de las que forma parte, pero sin embargo sí que percibe un valoración general de el mismo como persona.

\section{Modelo verdadero.}

Propuesto por Byrne (1996) parte de la premisa que la valoración general del autoconcepto debe obtenerse a partir de la suma de las autopercepciones generales del individuo y no sobre las autopercepciones específicas, referidas a dimensiones concretas. Este modelo parte de la definición que Rosenberg (1979) hace de la autoestima global. Entiende que todos los aspectos que la componen tienen la categoría suficiente para ser evaluados de forma diferente, por lo que en ningún momento está enfrentado a un modelo multidimensional del autoconcepto. Ahora bien, si rechaza el modelo nomotético ya que considera que la autoestima global no puede ser la suma de diferentes especificidades de la misma, ya que existen muchas influencias e interrelaciones que interfieren entre las diferentes dimensiones del autoconcepto y que producen variaciones importantes en dicha suma.

\section{Modelos multidimensionales.}

Son aquellos que parten de la premisa de que cada persona además de tener una valoración general de sí misma, también tienen una valoración concreta sobre cada uno de los ámbitos relevantes de los que forma parte, como el familiar, el social, el escolar, etc. (Cava, Musitu \& Vera, 2000). Partiendo de esta afirmación, el sujeto puede tener una valoración alta en un campo concreto, por ejemplo, el social, y baja en otro, como el académico. Estas valoraciones específicas de cada ámbito tampoco son equivalentes entre sí, aunque todas contribuyen en alguna medida a la percepción que cada uno tiene de su valía personal.

\section{Modelo de factores independientes.}

Planteado por Soares y Soares (1980), afirman que el autoconcepto está conformado por diferentes dimensiones que tienen una característica fundamental, no estar relacionadas entre sí, o poseer una correlación poco relevante. Por tanto, mantiene una postura opuesta al entender al autoconcepto como una construcción general o de una dimensión, a la vez que también se opone a entenderlo como un conjunto de facetas interrelacionadas significativamente.

\section{Modelo de factores correlacionados.}

Para este modelo las diferentes dimensiones que componen el autoconcepto presentan una alta correlación entre sí, pero además dicha correlación es igual de importante cuando se relaciona con la medida general del mismo (Harter, 1990). 


\section{Modelo compensatorio.}

El autoconcepto según este modelo estaría formado por una dimensión general y múltiples dimensiones de rango menor, que mostrarían una correlación negativa entre sí. Sin embargo, todas ellas aportarían diferente valor a la autovaloración general. El modelo es propuesto por Marx y Winne (1978) y lo definen como compensatorio porque entienden que, si una persona presenta una baja autovaloración en una dimensión concreta, por ejemplo, en la académica, la tendrá que compensar con una puntuación elevada en otra dimensión, por ejemplo la familiar.

Modelo taxonómico.

Propuesto por Soares y Soares (1977), y basado en el modelo de inteligencia planteado por Guilford (1969), plantean que el autoconcepto tiene al menos dos facetas, cada una de las cuales presenta al menos dos niveles. Esta estructura mantendría una correlación entre sus diferentes elementos que a su vez estarían organizados jerárquicamente.

\section{Modelo jerárquico.}

Aquí se encuentra un autoconcepto global y una serie de autoconceptos específicos, a un nivel inferior, que están separados, pero muestran cierta correlación entre sí. Por lo tanto, recoge determinados principios de los modelos anteriores, incluso de los unidimensionales, ya que plantea que en lo alto de la jerarquía existe un valor general del autoconcepto. Un autor representativo de este modelo es Epstein (1973), que propone un autoconcepto general y cuatro tipos de autoconceptos específicos de un nivel inferior: competencia, autoaprobación moral, poder y amor propio. Sin embargo, el modelo más representativo fue el diseñado por Shavelson, Hubner y Stanton (1976), donde además de un autoconcepto general, ubicado en lo alto de la jerarquía, aparecen una serie de autoconceptos de menor entidad como son el social, emocional, físico y académico. Cada uno de ellos además está dividido en otras especificidades de menor nivel, como por ejemplo el académico, que estaría basado en percepciones sobre la competencia personal en diferentes áreas del currículo.

\section{Autoconcepto en la educación.}

La educación en general es necesario que evolucione en la búsqueda de nuevas formas de trabajar de los profesores con los estudiantes. Es por ello la importancia del autoconocimiento que tenga los estudiantes en el transcurrir de su formación educativa, donde irán adquiriendo nuevos estilos y conceptos de sí mismo, los cuales percibir conjuntamente con los agentes de la institución educativa con quienes reforzaran dichos modelos adquiridos.

\section{Según Esnaola (2008):}

Una posible intervención educativa a través del auto concepto va a permitir acercarse en la medida de lo posible las visiones del mundo actual de los estudiantes, del adulto y educador, lo cual compromete que conozca mejor y valore los grupos sociales significativos del contexto en que estos se desenvuelven, al mismo tiempo que se trabajara sus valores para poder tener un resultado satisfactorio en su educación. (p. 48).

Naturalmente esto requiere de un conocimiento más profundo de la representación de entender la vida por parte de los juventud referente a sus actitudes y motivaciones dicha intervención se basaría en los siguientes presupuestos esenciales, lugar se incluye como variable eje de la personalidad de los estudiantes el auto concepto por ser el momento evolutivo en el que 
se está consolidando., se tiene en cuenta la importante influencia que ejerce el contexto social en que se desenvuelven lo cual va a suponer planificar diferentes formas de intervenir educativamente, en función de los grupos sociales de dicho contexto con la familia docente y pares. La educación en general es necesario que evolucione en la búsqueda de nuevas formas de trabajar profesores y alumnos con los actuales acordes de su visión del mundo, lo cual va a requerir de una formación específica.

De igual manera la posible intervención educativa debería contemplarse mediante un desarrollo de diferentes técnicas de trabajo que conlleve que el estudiante se sienta libre de trabajar y de mostrar su potencial tanto académico, físico, social y emocional, en definitiva dicho planteamiento facilitaría la integración de los estudiantes con su entorno social y académico y tener una visión más ajustada a realidad del mundo exterior en que vive y de sí mismo al mismo tiempo esto sería una forma de reforzar también una percepción positiva de su rendimiento académico y de su rol como estudiante.

En este sentido la educación tiene un papel predominante en los estudiantes en lo referente a lo académico cuya dimensión se interrelaciona con el auto concepto, al desarrollarse satisfactoriamente las capacidades de los estudiantes conjuntamente con el desarrollo de habilidades y destrezas, nos permite inferir que son estudiantes que han recibido estímulos positivos para lograr tener la seguridad emocional, por ende, se tendrá resultados favorables. Sin embargo, esta el otro lado de la moneda en cuanto estudiantes de un auto concepto bajo y no logran desarrollar en plenitud su rendimiento académico, esto se debe a que no son estimulados a temprana edad debido a muchos factores, investigaciones aportan que no es solo en la familia y sociedad, es juntamente con las instituciones educativas.

Por tanto, es de suma relevancia que, los estudiantes de las diversas instituciones educativas tengan en cuenta aquellos valores que interfieren negativamente, en su desarrollo ocasionando dificultades en la convivencia de los estudiantes teniendo dentro, de estas problemáticas de género y los modelos asociados a dichas diferencias como los estereotipos a seguir.

Esnaola (2008) precisó que el autoconocimiento requiere de un conocimiento más profundo de la forma de entender la vida por parte de los jóvenes referentes a sus actitudes y motivaciones dicha intervención se basaría en los siguientes presupuestos esenciales:

En primer lugar, se incluye como variable eje de la personalidad de los estudiantes el auto concepto por ser el momento evolutivo en el que se está consolidando. En segundo lugar, se tiene en cuenta la importante influencia que ejerce el contexto social en que se desenvuelven lo cual va a suponer planificar diferentes formas de intervenir educativamente, en función de los grupos sociales de dicho contexto con la familia docente y pares. En tercer lugar, la educación en general es necesario que evolucione en la búsqueda de nuevas formas de trabajar profesores y alumnos con los actuales acordes de su visión del mundo, lo cual va a requerir de una formación específica. En cuarto lugar, la posible intervención educativa debería contemplarse mediante un desarrollo de diferentes técnicas de trabajo que conlleva que el estudiante se sienta libre de trabajar y de mostrar su potencial tanto académico, físico, social y emocional ,en definitiva dicho planteamiento facilitaría la integración de los estudiantes con su entorno social y académico y tener una visión más ajustada a la realidad del mundo exterior en que vive y de sí mismo y al mismo tiempo esto sería una forma de reforzar también una percepción positiva de su rendimiento académico y de su rol como estudiante. (p. 82). 


\section{Dimensiones del autoconcepto.}

García y Musitu (2009) en sus estudios realizados del autoconcepto en adolescentes abarcaron estas dimensiones: Autoconceptos académicos, autoconceptos sociales, autoconceptos emocionales, autoconceptos familiares y autoconceptos físicos.

\section{Dimensión 1: Autoconcepto académico.}

Esta referida a la sensación que el individuo muestra de la calidad del desenvolvimiento de su propio papel, en el área que se desarrolla. El dominio expresa a dos contextos: el académico y el del área laboral. Por otro lado, el dominio se desarrolla en función a dos ejes: el primero está referido a las emociones que el individuo tiene del desempeño de sus funciones mediante sus tutores o superiores y, segundo, está referido a cualidades específicas valorativas principalmente en ese contexto.

Según García y Musitu (1999) esta dimensión se encuentra relacionada de manera positiva con el tema psico social, el rendimiento académico y de trabajo, tomar en cuenta la calidad de ejecutar la labor, se acepta y la valoración de los compañeros, el ser líder y la responsabilidad, y de forma negativa académico/laboral y el conflicto (p. 19).

Además, Machargo (1991) expresó que "las vivencias de situaciones de dificultad en el colegio garantizarán que el estudiante desarrolle un autoconcepto escolar de manera negativa y de esta manera se incrementen las probabilidades de que desarrolle un autoconcepto de manera negativa" (p. 56).

\section{Dimensión 2: Autoconcepto social.}

De acuerdo con los autores de la escala, el "autoconcepto social se relaciona de manera positiva con el ajuste psicosocial y con los beneficios psicosociales, en el rendimiento escolar y de trabajo, con la valoración y afecto de los profesores y los superiores, la valoración de los pares los valores universales y de manera negativa con las conductas disruptivas, la agresión y la depresión. (García \& Musitu, 1999).

\section{Dimensión 3: Autoconcepto emocional.}

Esta referida a la percepción del individuo de la situación emocional y de las respuestas a contextos particulares, con un nivel de compromiso e implicancias en su diario vivir. Este tema tiene dos bases de significancia; es así que la primera está referida a la opinión principal de sus emociones y la segunda está referida a contextos más particulares, donde al otro individuo es de un grado superior.

García y Musitu (1999) manifestaron que el autoconcepto emocional correlaciona de manera positiva con las habilidades sociales, el autocontrol, el sentimiento de bienestar y "la aceptación de los iguales; y negativamente, con la sintomatología depresiva, con la ansiedad, con el consumo de alcohol y drogas y con la pobre integración social en el aula y en el ámbito" laboral.

\section{Dimensión 4: Autoconcepto familiar.}

Este referido a la sensación que tiene el individuo de su implicancia y esta es participante e integradora en el entorno familiar. "El significado de este elemento se articula de forma en dos ejes. Esta referido específicamente al entorno familiar, que tiene que ver con las relaciones de los padres y como son: la confianza y el afecto que debe reinar en la familia. El segundo aspecto está 
referido al contexto familiar y el entorno del hogar y estas variables desarrolladas son formulados de forma positiva y las otras dos, se formulan negativamente y estas hacen mención al sentimiento de no estar involucrados y de no ser aceptado por los otros miembros familiares.

Este factor, que "es uno de los más importantes del autoconcepto, según García y Musitu (1999) correlaciona de manera positiva con el rendimiento escolar y laboral, con el tema psicosocial, "con el sentimiento de bienestar, con la integración escolar y laboral, con la conducta prosocial, con valores universalistas, y con la percepción de salud física y mental. También correlaciona negativamente con la sintomatología depresiva" a ansiedad y el consumo de drogas (García \& Musitu, 1999).

\section{Dimensión 5: Autoconcepto físico.}

Este aspecto está referido a la sensación que tiene el individuo de su aspecto físico y de su condición física. El factor gira en torno a dos ejes que son complementarios en su significado. El primero alude a la práctica deportiva en su vertiente social, física y de habilidad. El segundo hace referencia al aspecto físico. Un autoconcepto físico alto significa que la persona se percibe físicamente agradable, que se cuida físicamente y que puede practicar algún deporte adecuadamente" y con éxito.

El autoconcepto físico esta correlacionado positivamente con la opinión de salud, con el autocontrol y con la opinión de las personas que tienen del bienestar, con el rendimiento del deporte y con la motivación del logro y con la integración en los ámbitos sociales y académicas. En este caso el autoconcepto físico correlaciona de forma negativa con el desequilibrio académico, también presenta la ansiedad y, en un grado menor, con la presencia de dificultades con los pares (García \& Musitu, 1999).

\section{Método}

Dentro del paradigma positivista se asumió el enfoque cuantitativo, el método de la investigación hipotético-deductivo. La investigación que se realizó fue sustantiva, de diseño no experimental, de corte transversal descriptivo correlacional; se trabajó con una población de 122 adolescentes y la muestra seleccionada por muestreo no probabilístico intencional. Se extrajo 98 adolescentes de 14 a 17 años que desearon participar del estudio; la técnica fue la encuesta y el instrumento fue un cuestionario AF5 Auto concepto forma 5 MUSITU (1991), realizando ciertas modificaciones de acuerdo con el contexto por eso se realizó la validación con juicios de expertos. La confiabilidad del instrumento determinada por Alfa de Cronbach presentó una buena fiabilidad =0.937. En el análisis de datos se empleó el software SPSS 23, el cual permitió elaborar análisis descriptivo e inferencial, para la contratación de hipótesis se utilizó la prueba no paramétrica de regresión logística binaria.

\section{Resultados}

La contrastación de las hipótesis se probó mediante la regresión logística, debido a que según la prueba de normalidad de datos la variable y los factores no presentan normalidad en los datos, ya que su valor "p" es menor al valor de significación teórica $\alpha=.01$ 
Tabla 1.

Coeficientes de la regresión logística del auto concepto

\begin{tabular}{|c|c|c|c|c|c|c|c|}
\hline \multicolumn{8}{|c|}{ Variables en la ecuación } \\
\hline & & $\mathrm{B}$ & Error estándar & Wald & $\mathrm{gl}$ & Sig. & $\operatorname{Exp}(B)$ \\
\hline \multirow[t]{6}{*}{$\overline{\text { Paso } 1^{a}}$} & ND1 & 2.435 & .75 & 10.522 & 1 & .001 & 11.417 \\
\hline & ND2 & 1.490 & .70 & 4.523 & 1 & .033 & 4.436 \\
\hline & ND3 & 2.294 & .70 & 10.565 & 1 & .001 & 9.911 \\
\hline & ND4 & 20.193 & 5928,096 & .000 & 1 & .997 & 588250528.80 \\
\hline & ND5 & 37.291 & 10027.270 & .000 & 1 & .997 & $\begin{array}{c}1567591984688 \\
1660\end{array}$ \\
\hline & Constante & -86.922 & 19536.751 & .000 & 1 & .996 & .000 \\
\hline
\end{tabular}

En la tabla 1 se encontró que la dimensión autoconcepto emocional tiene una puntuación Wald $=10.565$ y p $=.001<\alpha$ : .01, influye significativamente a la predicción del autoconcepto. Por lo tanto, se acepta la hipótesis general de investigación, ya que la dimensión o factor que predomina es la dimensión autoconcepto emocional.

Tabla 2.

Coeficientes de la regresión logística de los indicadores en el interior de autoconcepto académico

\begin{tabular}{cccccccc}
\hline Variables en la ecuación & \multicolumn{7}{c}{} \\
\hline \multicolumn{1}{c}{} & $\mathrm{B}$ & Error estándar & Wald & $\mathrm{gl}$ & Sig. & Exp(B) \\
\hline Paso 1 $^{\mathrm{a}}$ & NI1 & 2.099 & 1.569 & 1.789 & 1 & .181 & 8.161 \\
& NI2 & 20.654 & 15494.368 & .000 & 1 & .999 & 932871965.024 \\
& NI3 & 5.555 & 1.315 & 17.857 & 1 & .000 & 258.654 \\
& NI4 & 2.081 & .967 & 4.632 & 1 & .031 & 8.016 \\
& NI5 & 2.783 & 1.16 & 5.756 & 1 & .016 & 16.172 \\
& Constante & -44.951 & 15494.369 & .000 & 1 & .998 & .00
\end{tabular}

a. Variables especificadas en el paso 1: NI1, NI2, NI3, NI4, NI5.

En la tabla 2 se encontró que el indicador 3 inteligencia, tiene una puntuación Wald = 17.857 y $\mathrm{p}=.000<\alpha: .01$, influye significativamente a la predicción del autoconcepto académico.

Tabla 3.

Coeficientes de la regresión logística de los indicadores en el interior del autoconcepto social

\begin{tabular}{|c|c|c|c|c|c|c|c|}
\hline \multicolumn{8}{|c|}{ Variables en la ecuación } \\
\hline & & $\mathrm{B}$ & Error estándar & Wald & $\mathrm{gl}$ & Sig. & $\operatorname{Exp}(B)$ \\
\hline \multirow[t]{4}{*}{$\overline{\text { Paso } 1^{\mathrm{a}}}$} & NI6 & 22.024 & 9896.480 & .000 & 1 & .998 & $\begin{array}{c}3672402230.71 \\
9\end{array}$ \\
\hline & NI7 & 5.049 & 1.22 & 17.137 & 1 & .000 & 155.871 \\
\hline & NI8 & 3.003 & .73 & 16.796 & 1 & .000 & 20.153 \\
\hline & Constante & -35.465 & 9896.481 & .000 & 1 & .997 & .00 \\
\hline
\end{tabular}


En la tabla 3 se encontró que el indicador 7 relaciones interpersonales, tiene una puntuación Wald $=17.137 \mathrm{y} \mathrm{p}=.000<\alpha: .01$, influye significativamente a la predicción del auto concepto social.

Tabla 4.

Coeficientes de la regresión logística de los indicadores en el interior del autoconcepto emocional

\begin{tabular}{llcccccc}
\hline \multicolumn{2}{l}{ Variables en la ecuación } & \multicolumn{7}{l}{} & & & & & \\
\hline Paso 1 $^{\text {a }}$ & NI9 & B & Error estándar & Wald & gl & Sig. & Exp(B) \\
& NI10 & 2.408 & .74 & 10.323 & 1 & .001 & 11.111 \\
& Constante & -44.764 & 7625.030 & .000 & 1 & .998 & 772109841.05 \\
& 15250.061 & .000 & 1 & .998 & .00
\end{tabular}

a. Variables especificadas en el paso 1: NI9, NI10.

En la tabla se encontró que el indicador 9 autoconfianza, tiene una puntuación Wald $=10.323$ y $\mathrm{p}=.000<\alpha: .01$, influye significativamente a la predicción del autoconcepto emocional.

Tabla 5.

Coeficientes de la regresión logística de los indicadores en el interior autoconcepto familiar

\begin{tabular}{|c|c|c|c|c|c|c|c|}
\hline \multicolumn{8}{|c|}{ Variables en la ecuación } \\
\hline & & B & Error estándar & Wald & $\mathrm{gl}$ & Sig. & $\operatorname{Exp}(\mathrm{B})$ \\
\hline \multirow[t]{6}{*}{$\overline{\text { Paso } 1^{\mathrm{a}}}$} & NI11 & 3.888 & 2.38 & 2.661 & 1 & .103 & 48.792 \\
\hline & NI12 & 2.789 & .98 & 8.037 & 1 & .005 & 16.266 \\
\hline & NI13 & 3.063 & 1.29 & 5.611 & 1 & .018 & 21.399 \\
\hline & NI14 & -20.409 & 28420.619 & .000 & 1 & .999 & .00 \\
\hline & NI15 & 23.919 & 28420.619 & .000 & 1 & .999 & 24434775362.5 \\
\hline & Constante & -21.819 & 6.18 & 12.451 & 1 & .000 & .00 \\
\hline
\end{tabular}

En la tabla 5 se encontró que el indicador 13 integración familiar, tiene una puntuación Wald $=5.611 \mathrm{y} \mathrm{p}=.018<\alpha: .05$, influye significativamente en el autoconcepto familiar.

Tablas 6.

Coeficientes de la regresión logística de los indicadores en el interior del autoconcepto físico

\begin{tabular}{|c|c|c|c|c|c|c|c|}
\hline \multicolumn{8}{|c|}{ Variables en la ecuación } \\
\hline & & B & Error estándar & Wald & $\mathrm{gl}$ & Sig. & $\operatorname{Exp}(\mathrm{B})$ \\
\hline \multirow[t]{4}{*}{$\overline{\text { Paso } 1^{\mathrm{a}}}$} & NI16 & 3.252 & .733 & 19.709 & 1 & .000 & 25.853 \\
\hline & NI17 & -2.686 & 1.147 & 5.485 & 1 & .019 & .068 \\
\hline & NI18 & 2.502 & .695 & 12.975 & 1 & .000 & 12.203 \\
\hline & Constante & -6.648 & 1.637 & 16.488 & 1 & .000 & .001 \\
\hline
\end{tabular}


En la tabla se encontró que el indicador 16 Participación deportiva, tiene una puntuación Wald $=3.252$ y $\mathrm{p}=.000<\alpha: .01$, influye significativamente en el autoconcepto.

\section{Discusión}

El estudio presento seis objetivos en relación al Objetivo general (Determinar la dimensión predominante que más influye en el autoconcepto en estudiantes con problemas sociales). El factor predominante de esta investigación es el autoconcepto emocional, esto debido a que según nos muestra sus dimensiones ante una percepción baja de autoconcepto emocional. Al respecto Chávez (2016) en el trabajo de investigación concluye que de los 151 estudiantes que fue su muestra, el 53\% manifiestan un nivel medio, mientras que el 39.7\% indican un nivel alto y el $7.3 \%$ un nivel bajo con respecto a la variable autoconcepto. Respecto a cada una de los componentes del autoconcepto fueron de los 151 estudiantes, el 53.6\% manifiestan un nivel alto, mientras que el $41.7 \%$ indican un nivel medio y el 4,6\% un nivel bajo con respecto a la dimensión autoconcepto académico, el $51.7 \%$ manifiestan un nivel medio, mientras que el $43 \%$ indican un nivel alto y el 5.3\% un nivel bajo con respecto a la dimensión autoconcepto social, el 53\% manifiestan un nivel medio, mientras que el 33,8\% indican un nivel alto y el $13.2 \%$ un nivel bajo con respecto a la dimensión autoconcepto emocional, el $47 \%$ manifiestan un nivel alto, mientras que el $46.4 \%$ indican un nivel medio y el $6,6 \%$ un nivel bajo con respecto a la dimensión autoconcepto familiar y el 47,7\% manifiestan un nivel alto, mientras que el $45 \%$ indican un nivel medio y el $7.3 \%$ un nivel bajo con respecto a la dimensión autoconcepto físico.

El indicador predominante dentro de la dimensión autoconcepto académico es el indicador inteligencia, coincidiendo con la investigación de Martínez y Mañas (2014) concluye la existencia de una tendencia general que indicaría que a medida que aumenta el autoconcepto académico aumenta el rendimiento académico. Los alumnos que son competitivos en lo académico son también los que tienen una imagen positiva a nivel físico y se sienten aceptados en su grupo de iguales. Desaparece cada vez más el estereotipo del clásico "empollón”, con una imagen descuidada y socialmente no aceptado. Según los datos de nuestra investigación, los alumnos que se consideran buenos académicamente son también los que piensan que su aspecto físico es adecuado y que son habilidosos en sus relaciones con el grupo de iguales como apoyan investigaciones (García \& Musitu, 2009).

El indicador predominante dentro de la dimensión autoconcepto social es relaciones interpersonales. Al respecto Gómez (2010) concluye que el auto concepto es muy importante para la salud mental y el buen desarrollo afectivo de los estudiantes. Entendiendo al "auto concepto como el conocimiento que la persona tiene conscientemente acerca de sí mismo, o también, es la imagen que tenemos de nosotros.

El indicador predominante dentro de la dimensión autoconcepto emocional es el indicador autoconfianza. Al respecto La teoría del aprendizaje social de Bandura (1986) quien manifestó que es esta a través de esta teoría se forma el autoconcepto. Los niños aprenden comportamientos, actitudes, movimientos, etc. a través de la observación. Tienden a imitar la conducta de aquellas personas que son relevantes y queridas para él (los otros significativos), y por tanto son sus modelos a seguir. Estos son los familiares cercanos, en primer momento, y los personajes del ámbito escolar, posteriormente. A través del proceso de imitación, el niño va haciendo suyas las conductas y actitudes observadas que son de su agrado hasta incorporarlas en sus propios esquemas llegando a identificarlas, posteriormente, como características personales. 
El indicador predominante dentro de la dimensión autoconcepto familiar es el indicador integración familiar. Coincidiendo con Huarhua (2016), en el presente estudio los resultados obtenidos, revelaron en la estadística descriptiva que de 200 estudiantes, el $13 \%$ presentan un nivel bajo, el $63 \%$ presentan nivel medio y el $24 \%$ de ellos presentan un nivel alto de autoconcepto siendo el autoconcepto familiar el más representativo con un $49 \%$ de nivel, para la muestra encontrándose con un $49 \%$ y para la constrastación de hipótesis se realizó el modelo de regresión es estadísticamente significativa, $\mathrm{X} 2=16.455 ; \mathrm{p}=.012<.05$ y el modelo explica el $9.5 \%$ (R2 de Nagelkerke) se debe a la edad y autoconcepto, por lo tanto se toma la decisión de aceptar la hipótesis alterna, es decir: La edad influye en el autoconcepto.

El indicador predominante dentro de la dimensión autoconcepto físico es el indicador Participación deportiva. Los resultados coinciden con el estudio de Véliz (2010) quien concluye que a nivel de autoconcepto físico los estudiantes universitarios hombres presentan mayor nivel de autoconcepto Emocional y Físico, elementos a tener en cuenta cuando se trabaja con los estudiantes. Se aprecia además que en la medida que los estudiantes tienen mayor edad mejoran sus niveles de autoconcepto académico, emocional, familiar y físico, por lo que el énfasis debe estar en el trabajo con los estudiantes más noveles.

\section{Referencias}

Bandura, A. (1986). Social foundations of thought and action: a social cognitive theory. Prentice Hall, Englewood Cliffs, NJ: Prentice Hall.

Belart, A., \& Ferrer, M. (1999). El ciclo de la vida. Una visión sistémica de la familia. Bilbao: Descleé de Brouwer.

Bernal, C. (2006). Metodología de la investigación. México: Pearson Educación de México. Bisquerra, R. (1998). Modelos De Orientación e Intervención Psicopedagógica. Barcelona: Praxis. Boersma, F. y Chapman, J. (1992). La percepción de la Escala Capacidad para estudiantes: Manual.Los Ángeles: Western Psychological Services.

Bracken, B. (1992). Escala de Autoconcepto multidimensional. Austin, TX: Pro-Ed.

Branden, N. (1993). El poder de la autoestima. Cómo potenciar este importante recurso psicológico. Barcelona: Paidós.

Byrne, B. (1996). La medición de autoconcepto durante toda la vida. Problemas e instrumentación. Washington: APA.

Byrne, D., \& Baron, R. (2005). Psicología Social. México: Pearson Educación.

Cardenal, V. (1999). El Autoconocimiento y la Autoestima en el Desarrollo de la Madurez Personal. Málaga: Aljibe.

Carretero, M. (1997). Introducción a la Psicología Cognitiva. Buenos Aires: Aique.

Cava, M., Musitu, G., \& Vera, A. (2000). Intervención psicosocial en adolescentes con problemas deintegración social. Psicología y Salud, 10(2), 215-226. Recuperado de: http://go.galegroup.com/ps/anonymous?id=GALE\%7CA116037453\&sid=googleScholar\& $\mathrm{v}=2.1 \& \mathrm{it}=\mathrm{r} \&$ linkaccess $=$ abs\&issn $=14051109 \& \mathrm{p}=\mathrm{AONE} \& \mathrm{sw}=\mathrm{w}$

Cerviño, C. (2008). Autoestima y desarrollo personal. Influencia en el rendimiento escolar. Módulo del Programa de Doctorado 700H Psicología de la Educación y Desarrollo Humano de la Universitat de València. Lima: ISPP Crea.

Chávez, P.G. (2016). Autoconcepto y las habilidades sociales de los estudiantes de la experiencia curricular de filosofía de la escuela de Administración de la Universidad César Vallejo - San Juan de Lurigancho, 2015 (Tesis de maestría). Universidad César Vallejo, Lima.

Contreras, V. (2010). Autoconcepto y rendimiento Matemático en estudiantes de $4^{\circ}$ y $5^{\circ}$ grado de secundaria de una institución educativa de Ventanilla (Tesis de maestría). Universidad San Ignacio de Loyola, Lima.

Coopersmith, S. (1967). Los antecedentes de la autoestima. San Francisco: W. H. Hombre libre.

Droguett, L. (2011). Rasgos psicológicos asociados al ajuste social y personal de alumnado adolescente. Recuperado de: 
https://addi.ehu.es/bitstream/handle/10810/12202/DROGUETT\%20LATORRE.pdf;jsessi onid=D298E5776326EEC715388CEFAEC06538? sequence $=1$

Epstein, S. (1973). El concepto de sí mismo o de una teoría revisada de una teoría. American Psychologist, 28(1), 405-416. Recuperado de: http://psicologos.mx/2-el-concepto-del-simismo.php

Esnaola, I. (2008). El autoconcepto físico durante el ciclo vital. Anales de Psicología, 24(1), 1-8.

Recuperado de: https://www.redalyc.org/html/167/16724101/

Fournier, C. (2004). Comunicación Verbal. México: Thomson Learning Ibero-México.

García, A. (1999). Autoconcepto y necesidades educativas especiales en el sector educativo de Brozas (Cáceres). Revista de Educación, 318(1), 251-269.

García, F., \& Musitu, G. (1999). AF5. Autoconcepto Forma 5 Manual. España: Universidad de Valencia. TEA Ediciones.

Gómez, L. (2010). Clima escolar social y autoconcepto en alumnos de educación secundaria de Lima (Tesis de maestría). Universidad Nacional Mayor de San Marcos, Lima.

Goñi, A. (2009). El autoconcepto físico. Madrid: Pirámide.

Guilford, J. (1969). La naturaleza de la inteligencia humana. New York: McGraw-Hill.

Harter, S. (1990). Problemas en la evaluación del autoconcepto de los niños y adolescentes. Cognitive Therapy and Research, 14(2), 113-142.

Hidalgo, C., \& Abarca, N. (2000). Comunicación Interpersonal. Programa de entrenamiento en habilidades sociales. Santiago de Chile: Universidad Católica de Chile.

Huarhua, R. M (2016). Factores asociados al autoconcepto con sus dimensiones de los estudiantes universitarios de Lima 2016 (Tesis de maestría). Universidad César Vallejo. Lima.

Kimmel, D., \& Weiner, I. (1998). La adolescencia: una transición del desarrollo. Barcelona: Ariel Psicología.

Machargo, J. (1991). El profesor y el autoconcepto de sus alumnos: teoría y práctica. Madrid: Escuela Española.

Madrigales, C. (2012). Autoconcepto en adolescentes de 14 a 18 años (Tesis de Licenciatura). Universidad Rafael Landívar, Quetzaltenango, México.

Marsh, H., \& Hattie, J. (1996). Theoretical perspectives on the structure of selfconcept. Handbook of Self-Concept: Developmental, social and clinical considerations. New York: Wiley.

Martínez, A., \& Mañas, C. (2014). Autoconcepto y rendimiento académico en adolescentes. Revista International Journal of Developmental and Educational Psychology, 1(2), 555-564. Recuperado de: http://dehesa.unex.es/handle/10662/1641

Marx, R., \& Winne, P. (1978). Construir interpretaciones de tres inventarios de autoconcepto. American Educational Research Journal, 15(1), 99-108.

Mingote, J., \& Requena, M. (2008). El malestar de los jóvenes: contextos, raíces y experiencias. Madrid: Díaz de Santos.

Palmer \& Alberti (1992). La asertividad. Barcelona: Descle.

Pérez, N. (2014). Autoconcepto en estudiantes de 13 a 16 años de familias integradas y desintegradas del instituto de educación básica por Cooperativa El Esfuerzo (Tesis de Licenciatura). Universidad de San Carlos de Guatemala, Guatemala.

Picard, Ch. (2002). Mediación en conflictos interpersonales y de pequeños grupos. La Habana: Publicaciones Acuarios. Centro Félix Valera.

Piers, E., \& Harris, D. (1964). Age and other correlates of self-concept in children. Journal of Educational Psychology, 55(1), 91-95. Recuperadode: http://psycnet.apa.org/record/196501354-001

Powel, B. (2005). Las relaciones personales. Clave de salud. México: Urano.

Roca, M. (2003). Conflicto y vida cotidiana. Buenos Aires: Infomed con PHP-Nuke.

Roid, G., \& Fitts, W. (1988). Tennessee escala de auto-concepto. Los Ángeles: Western Psychological Services.

Rosenberg, M. (1979). Concebir el yo. Nueva York: Basic Books.

San Martin, V., \& Andrade, M. (2000). La Transversalidad en la Educación Chilena. Talca: Instituto del Valle Central.

Sánchez, H., \& Reyes C. (2015). Metodología y Diseños en la Investigación Científica. Editorial Business Support Aneth S.R.L 
Schmidt, A. (2010). Autoconcepto y Motivaciones Vocacionales en estudiantes de primer año de carreras de Humanidades y Ciencias Exactas (Tesis de maestría). Pontificia Universidad Católica Argentina, Paraná.

Shavelson, R. J., Hubner, J. J., \& Stanton, J. C. (1976). Self concept:validation of construct interpretations. Review of educational Research, 46, 407-441. Doi: https://doi.org/10.3102/00346543046003407

Soares, A., \& Soares, L. (1980). Manual de la prueba: El Inventario de Percepción afectivo. Trumbull, CT: Soares Associates.

Soares, L., \& Soares, A. (1977). El concepto de sí mismo: mini, maxi, múltiples. Nueva Jersey: Documento presentado en la reunión anual de la American Educational Research Association.

Soares, L., \& Soares, A. (1983). Los componentes de auto-cogniciones relacionadas con los estudiantes. Montreal: Trabajo presentado en la Reunión Anual de la Asociación Americana de Investigación Educativa, de Montreal.

Sosa, D. (2014). Relación entre autoconcepto, ansiedad e inteligencia emocional: eficacia de un programa de intervención en estudiantes adolescentes (Tesis doctoral). Universidad de Extremadura, España.

Véliz, A. (2010). Dimensiones del autoconcepto en estudiantes chilenos: Un estudio psicométrico. (Tesis Doctoral). Universidad del País Vasco, Bilbao. 